\title{
An optimum city size? The scaling relationship for urban population and fine particulate $\left(\mathrm{PM}_{2.5}\right)$ concentration
}

\author{
Lijian Han a, Weiqi Zhou ${ }^{\text {a, }}{ }^{*}$, Steward T.A. Pickett ${ }^{b}$, Weifeng $\mathrm{Li}^{\mathrm{a}}$, $\mathrm{Li} \mathrm{Li}^{\mathrm{c}}$ \\ a State Key Laboratory of Urban and Regional Ecology, Research Center for Eco-Environmental Sciences, Chinese Academy of Sciences, Beijing 100085, China \\ ${ }^{\mathrm{b}}$ Cary Institute of Ecosystem Studies, Millbrook, NY 12545-0129, United States \\ ' School of Urban Planning and Design, Peking University Shenzhen Graduate School, Shenzhen 518055, China
}

\section{A R T I C L E I N F O}

\section{Article history:}

Received 30 March 2015

Received in revised form

12 August 2015

Accepted 21 August 2015

Available online 23 October 2015

\section{Keywords:}

Urbanization

Developed country

Developing country

Urban air quality

\begin{abstract}
A B S T R A C T
We utilize the distribution of $\mathrm{PM}_{2.5}$ concentration and population in large cities at the global scale to illustrate the relationship between urbanization and urban air quality. We found: 1) The relationship varies greatly among continents and countries. Large cities in North America, Europe, and Latin America have better air quality than those in other continents, while those in China and India have the worst air quality. 2) The relationships between urban population size and $\mathrm{PM}_{2.5}$ concentration in large cities of different continents or countries were different. $\mathrm{PM}_{2.5}$ concentration in large cities in North America, Europe, and Latin America showed little fluctuation or a small increasing trend, but those in Africa and India represent a " $U$ " type relationship and in China represent an inverse " $U$ " type relationship. 3) The potential contribution of population to $\mathrm{PM}_{2.5}$ concentration was higher in the large cities in China and India, but lower in other large cities.
\end{abstract}

(๑) 2015 Elsevier Ltd. All rights reserved.

\section{Introduction}

The rapid urbanization in the last century has resulted in more than half of the world's population living in cities (Population Division, 2012). The population in cities, particularly in large cities, could generate intensive human social and economic activities that result in urban environmental pollutions (e.g. air pollution). Therefore, special concern has been given to the effect of global urbanization, particularly increase in population and associated activities on air quality. Urban air pollution is highly attributed to the fossil fuel consumption; however, energy consumption efficiency, and per capita emission differ among continents and countries (Mayer, 1999; Lamsal et al., 2013). A few studies have taken limited traditional air pollutants (e.g. $\mathrm{NO}_{2}$ ) as indicator to illustrate the relationship between urban air pollution and urban population size, because $\mathrm{NO}_{2}$ has a short lifetime and can be predominantly attribute to local sources (Lamsal et al., 2013). However, other major air pollutants, such as fine particulate matter $\left(\mathrm{PM}_{2.5}\right)$, have not been well examined and documented. Our objective in this paper is therefore to investigate the relationship between urban air quality in terms of $\mathrm{PM}_{2.5}$ concentration, and urban

\footnotetext{
* Corresponding author.

E-mail addresses: ljhan@rcees.ac.cn (L. Han), wzhou@rcees.ac.cn (W. Zhou).
}

population size in global large cities. This paper will document the variety of relationships between urban ambient air pollution represented by $\mathrm{PM}_{2.5}$, and explore the specific patterns that exist in different regions and countries.

$\mathrm{PM}_{2.5}$ is one of major air pollutants in many large cities, and is a toxic component that threatens the public health (Pope et al., 2006; Han et al., 2015a,b). A number of developed countries (e.g. United States) have established monitoring networks for $\mathrm{PM}_{2.5}$ measurement, while few developing countries (e.g. China), which are suffering from the most severe $\mathrm{PM}_{2.5}$ pollution, have built monitoring networks beyond limited stations (Han et al., 2014). Those networks provide accurate monitoring results at the specific locations. However, the utility of the data are limited by the number of the stations and the difficulty of quantitatively documenting the spatial pattern of $\mathrm{PM}_{2.5}$ concentration. Thus, the satellite derived $\mathrm{PM}_{2.5}$ concentration was utilized in this work to present a spatially exhaustive quantitative analysis (van Donkelaar et al., 2010).

Urban population size is a typical urbanization indicator of urban social and economic activities (e.g. fossil fuel combustion). Urban air pollution is confidently attributed to urban population size and its related activities (Lamsal et al., 2013). This research therefore utilized the global full-covered $\mathrm{PM}_{2.5}$ concentration to quantitatively investigate the scaling relationship between $\mathrm{PM}_{2.5}$ and urbanization in large cities at the global scale. 


\section{Materials and methodology}

\subsection{Large cities}

Large cities in this research are those with population size of more than 0.75 million and urban area of more than $100 \mathrm{~km}^{2}$ (Fig. 1). The population records in 2005 were obtained from the World Urbanization Prospects (available at http://esa.un.org/ unup/), and urban size was estimated from Moderate Resolution Imaging SpectroRadiometer (MODIS) global map of urban extent (Schneider et al., 2009, 2010). Based on the population and area criteria above, 381 large cities were selected: 162 in Asia, 76 in Europe, 55 in Latin America, 55 in North America, 29 in Africa, and 4 in the remainder of the globe. Moreover, because big cities in Asia were mainly at China (66) and India (33), we therefore examined the relationship between population amount and $\mathrm{PM}_{2.5}$ concentration to indicate Asia's general condition.

\section{2. $P M_{2.5}$ concentration in the large cities}

The global $\mathrm{PM}_{2.5}$ concentration dataset has a spatial resolution of $10 \mathrm{~km}$, as an annual average during 2001-2006 to meet with the similar time scale to the urban population and extension data (van Donkelaar et al., 2010). The dataset was derived from the combination of MODIS and Multi-angle Imaging SpectroRadiometer (MISR) aerosol optical depth with aerosol vertical profile and scattering properties, based on a simulation of the GEOS-Chem chemical transport model with a reasonable accuracy when comparing with ground measurements $(r=0.83$; slope $=0.86$; $\mathrm{n}=244$ ). And, the mean $\mathrm{PM}_{2.5}$ concentration in the large cities was then summarized by city with the $\mathrm{PM}_{2.5}$ concentration dataset and the global large cities' layer.

\subsection{Statistical analysis}

To examine how $\mathrm{PM}_{2.5}$ concentration varied by urban population size, we divided the cities into seven groups based on the population size of, $0.75-1,1-2,2-3,3-4,4-5,5-10$, and larger than 10 million. In addition, large cities with a population of more than 5 million were examined apart from the group of smaller cities. Correlation analysis was carried out between the mean $\mathrm{PM}_{2.5}$ concentration of the large cities and their population size, to explore the impact of urbanization on $\mathrm{PM}_{2.5}$ pollution.

\subsection{Potential contribution of urban population to $P M_{2.5}$ concentration}

We assumed that the urban $\mathrm{PM}_{2.5}$ concentration comes from the human activities in each city, and therefore take the $\mathrm{PM}_{2.5}$ concentration per capita as the potential contribution of urban population to $\mathrm{PM}_{2.5}$ concentration.

\section{Results}

$\mathrm{PM}_{2.5}$ concentration in the large cities varied among continents (Fig. 2A). $\mathrm{PM}_{2.5}$ concentration in $23.9 \%$ of the large cities was higher than the World Health Organization interim target-1 (Annual mean $\mathrm{PM}_{2.5}$ concentration less than $35 \mu \mathrm{g} / \mathrm{m}^{3}$; IT- 1 ), and only $18.0 \%$ of large cities were within the World Health Organization air quality guidelines (Annual mean $\mathrm{PM}_{2.5}$ concentration less than $10 \mu \mathrm{g} / \mathrm{m}^{3}$; AQG). Large cities in Asia ranked the worst in $\mathrm{PM}_{2.5}$ concentration at the global scale: $48.7 \%$ of its large cities had $\mathrm{PM}_{2.5}$ concentration higher than the IT- 1 , and only $1.7 \%$ of its large cities had $\mathrm{PM}_{2.5}$ concentrations within the AQG. 6.8\% of large cities in Africa had $\mathrm{PM}_{2.5}$ concentration higher than the IT- 1 , and $27.3 \%$ of its large cities had $\mathrm{PM}_{2.5}$ concentration within the AQG. No $\mathrm{PM}_{2.5}$ concentration higher than the IT-1 was observed in large cities in North America and Europe. Large cities in Latin America had the best air quality with $64.4 \%$ of them possessing $\mathrm{PM}_{2.5}$ concentrations within the AQG.

Meanwhile, $\mathrm{PM}_{2.5}$ concentration in the large cities was also found to be variable among countries (Fig. 2B). In general, large cities in developed countries had better air quality than in those in developing countries. $31.8 \%$ of large cities in developing countries had $\mathrm{PM}_{2.5}$ concentration higher than the IT-1, whereas, no large cities in developed countries were found to have $\mathrm{PM}_{2.5}$ concentration higher than the IT-1. Large cities in the two fast-growing developing countries, China and India, were observed to have the worst air quality. For example, $78.5 \%$ of the large cities in China and

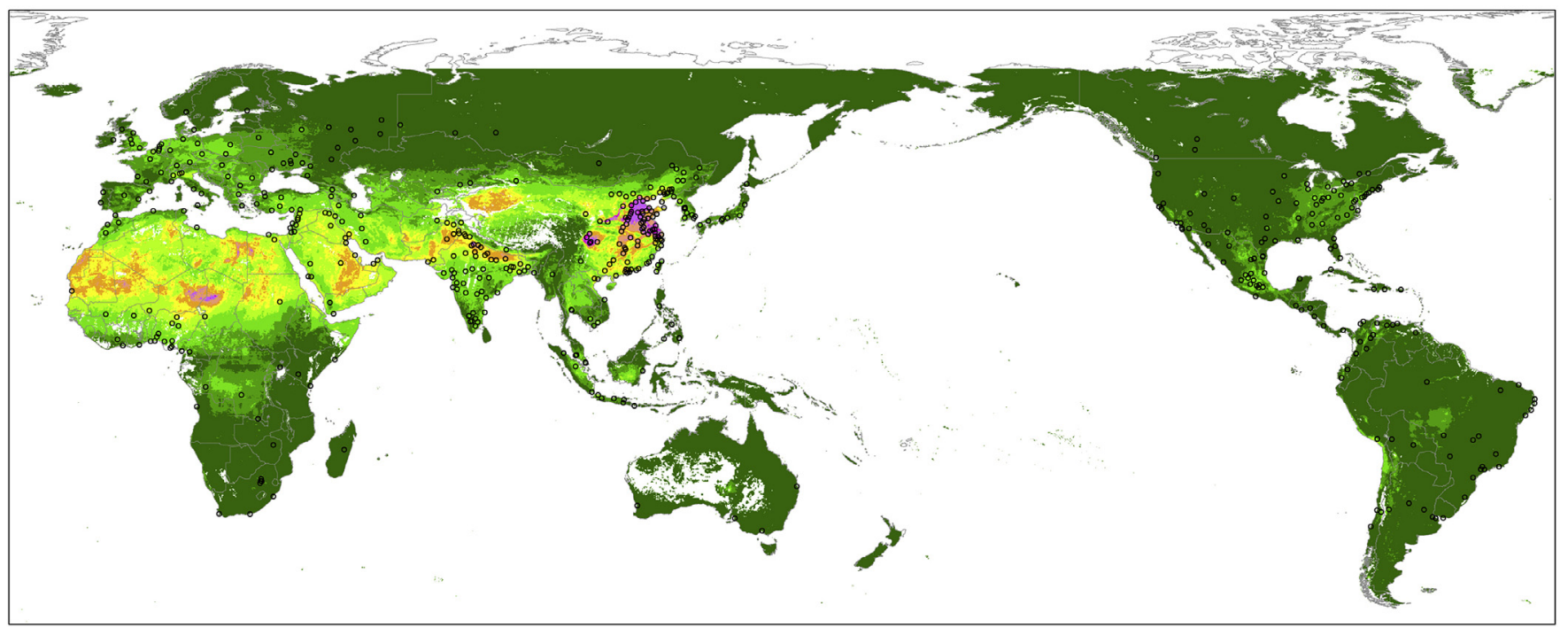

Large cities that analyzed in this work

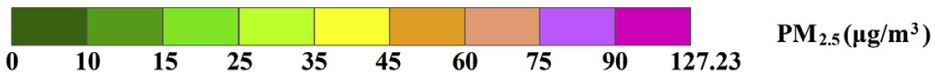

Fig. 1. Global large cities and $\mathrm{PM}_{2.5}$ concentration that used in this research. 


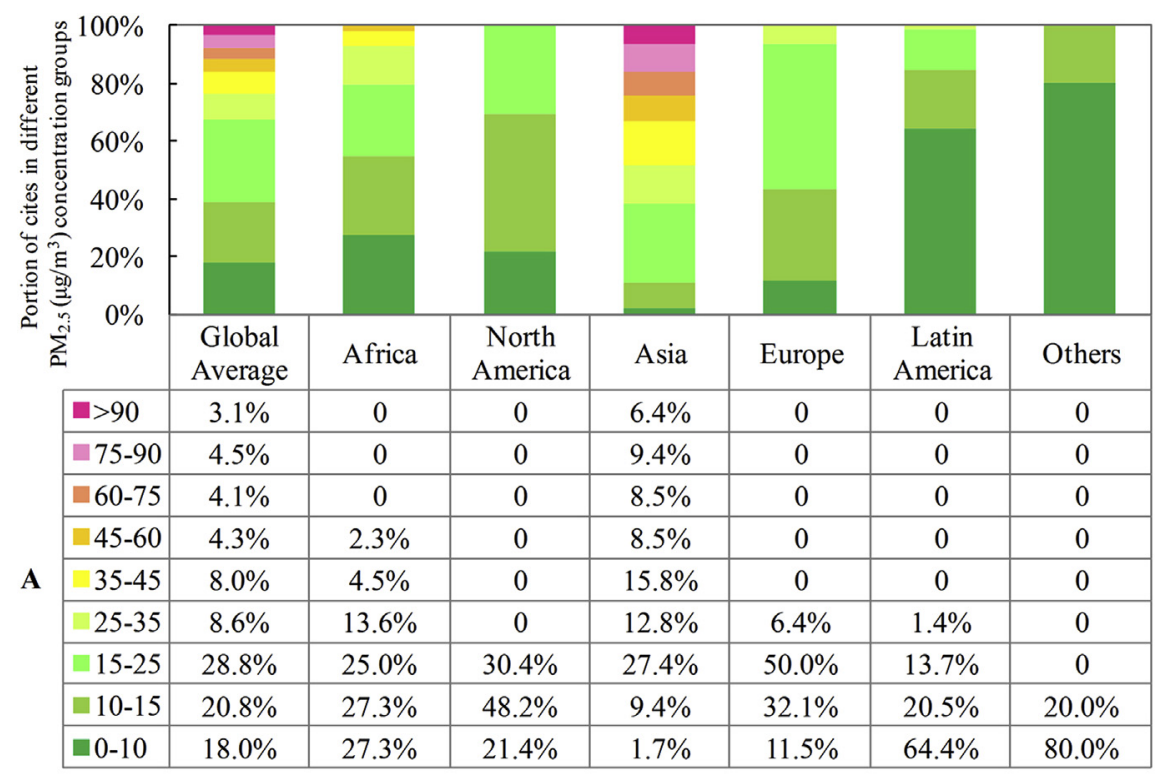

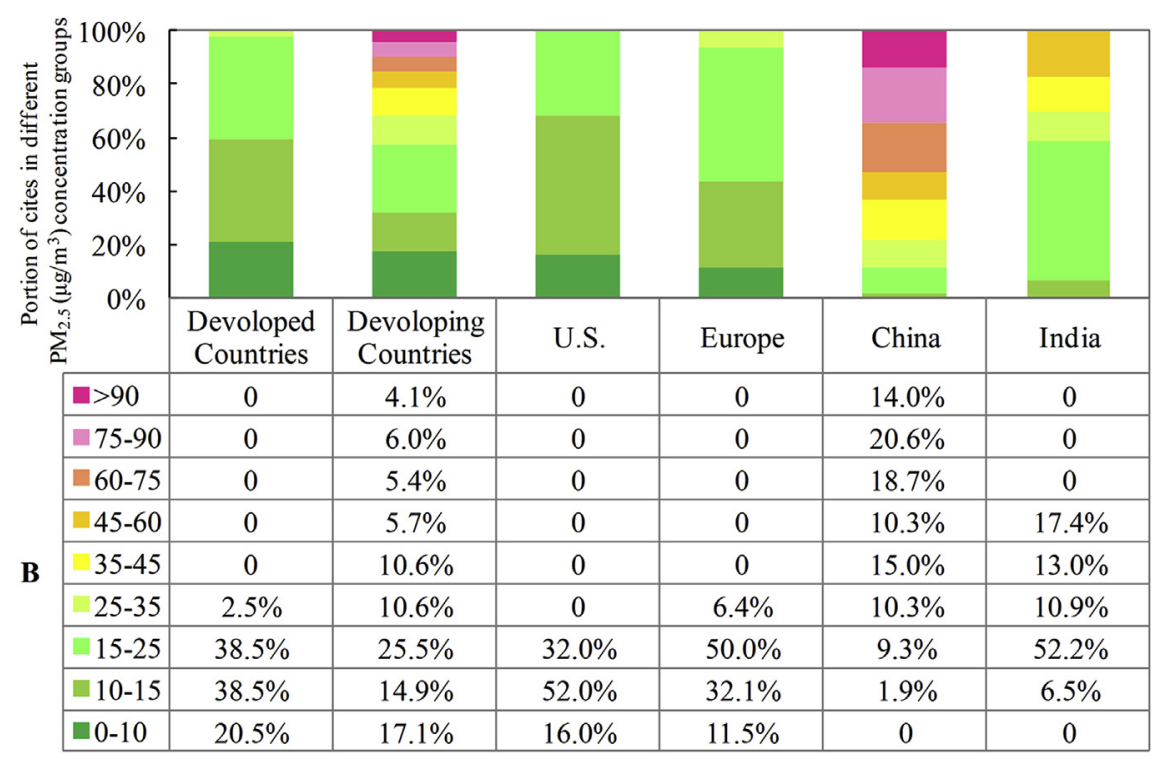

Fig. 2. Portion of large cities in different $\mathrm{PM}_{2.5}$ concentration groups at global/continent (A) and country/regional scales (B).

$30.4 \%$ of the large cities in India had $\mathrm{PM}_{2.5}$ concentrations larger than the IT-1, and none of them were within AQG. In contrast, large cities in Europe and North America were found with $\mathrm{PM}_{2.5}$ concentration within the IT- 1 and World Health Organization interim target-2 (Annual mean $\mathrm{PM}_{2.5}$ concentration less than $25 \mu \mathrm{g} / \mathrm{m}^{3}$; IT$2)$, respectively.

Diverse relationships between population size and $\mathrm{PM}_{2.5}$ concentration in large cities were obtained in different global areas (Fig. 3). Large cities in North America and Europe were found to have more or less stable or small increases in $\mathrm{PM}_{2.5}$ concentration with urban population size increase. A similar relationship was also observed in Latin America, however $\mathrm{PM}_{2.5}$ concentration in large cities with population more than 5 million showed a " $U$ " type trend with the increase of urban population size, while an inverse " $U$ " type trend and no significant trends were observed in European and North American large cities with population more than 5 million. $\mathrm{PM}_{2.5}$ concentration in large cities in Africa and India showed a " $U$ " type trend against the urban population size increase, and showed a significantly increasing trends in large cities with population more than 5 million (Africa: $\mathrm{R}^{2}=0.71, \mathrm{P}<0.05$; India $\left.\mathrm{R}^{2}=0.54, \mathrm{P}<0.05\right)$. An inverse " $\mathrm{U}$ " type relationship between $\mathrm{PM}_{2.5}$ concentration and urban population was observed in China $\left(\mathrm{R}^{2}=0.90, \mathrm{P}<0.05\right)$, and a significantly decreasing trend was observed at large cities with population more than 5 million $\left(\mathrm{R}^{2}=0.35, \mathrm{P}<0.05\right)$.

The potential population contribution to $\mathrm{PM}_{2.5}$ concentration showed a decrease against urban population size increase. Moreover, the potential contribution of population to $\mathrm{PM}_{2.5}$ concentration was higher in most large cities of China and India, as well as large cities in Africa, particularly those with population size from 0.75 to 1 million. The population's contribution to $\mathrm{PM}_{2.5}$ were diverse between different population size categories, and the largest gap of the contribution was particular diverse of developing countries/continents. In China, the largest gap between the contributions was found between population size of $1-2$ and 3-4 million, while this gap was found in large cities with population size between 3-4 and 4-5 million in India, and in large cities between $0.75-1$ and $1-2$ million in Africa. 

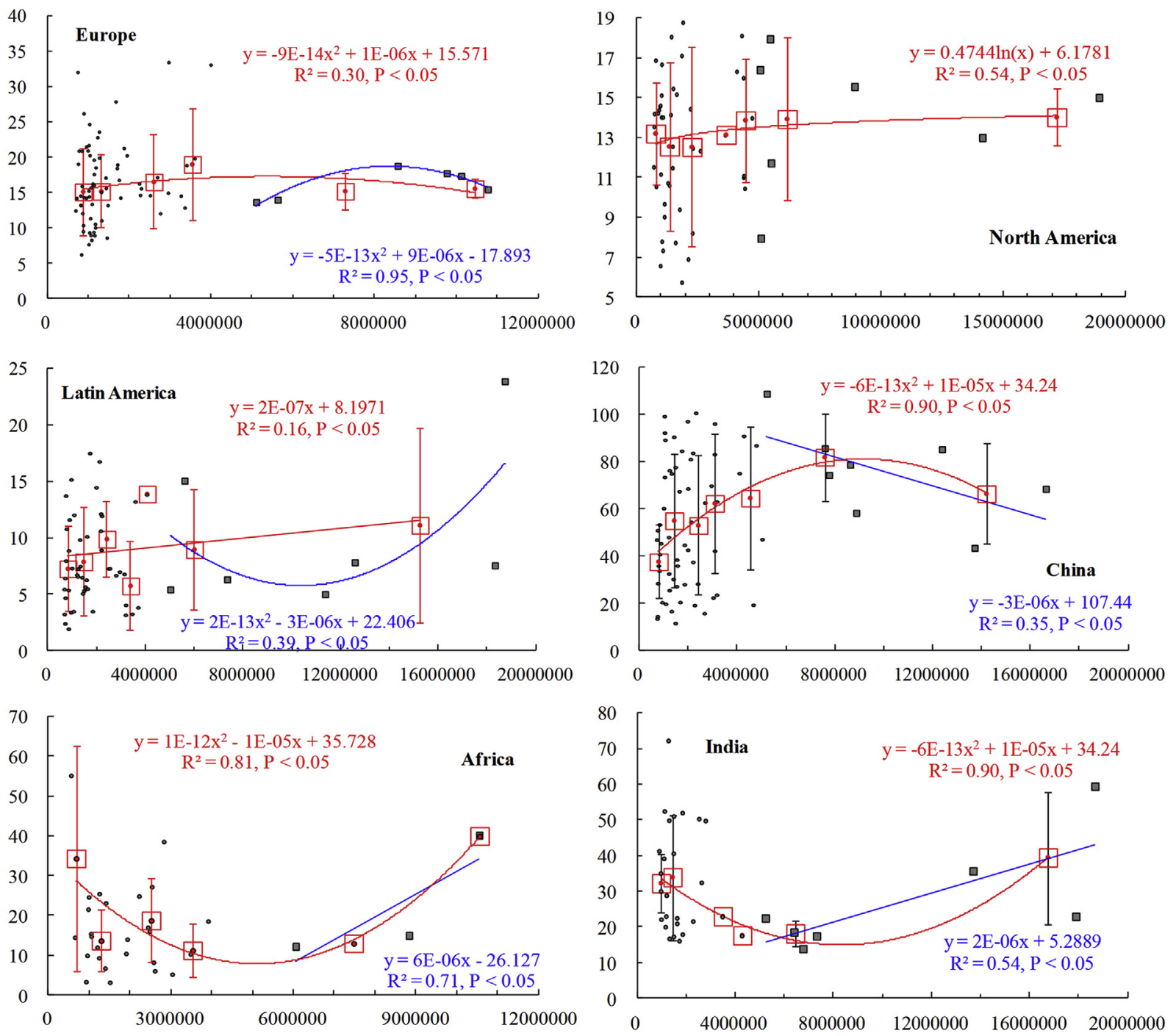

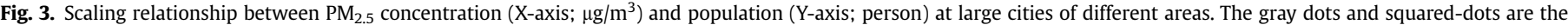

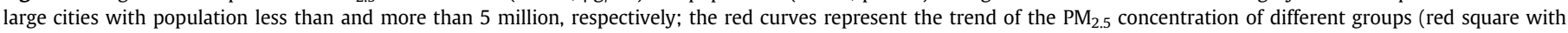

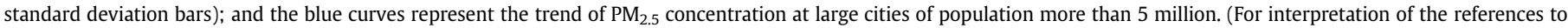
color in this figure legend, the reader is referred to the web version of this article.)

\section{Discussion}

The increase of urban population does not necessarily lead to serious degeneration of air quality in large cities in the developing countries. Generally, urban population size increase would enhance the magnitude of human activities that will directly or indirectly bring environmental problems, including severe air pollution in large cities at developing countries (Fenger, 2009; Soubbotina, 2004; Vitousek et al., 1997). We found large cities in China and India are suffering from negative impacts of urbanization. In contrast, large cities in North America and Europe had positive impact from urbanization. This is largely attributed to the country's technology and pollution abatement measures, particularly that in energy consumption (National Academy of Engineering and National Research Council (2007)). Using cleaner fossil fuels more efficiently and increasing the reliance on cleaner renewable energy sources are among the best ways to control and reduce air pollution without compromising economic growth (Soubbotina, 2004). In addition, energy structure and efficiency are diverse among countries. For example, $74.2 \%$ of China's electricity comes from coal, one of the "dirtiest" energy source, but $55.1 \%$ and $16.8 \%$ for USA and Russia, respectively. Therefore, the diversity of $\mathrm{PM}_{2.5}$ concentration in large cities of different areas could illustrate the energy structure and efficiency of fossil fuel utilization. Moreover, large cities in Latin America where also had many developing countries, however had positive impact from urbanization due to their trick control of pollutant emission and changing to cleaner fuels since the end of 1980s. Thus, further research is suggested to carry out more comprehensive analysis through combining urbanization, energy source and efficiency, and urban air quality, for better understanding the mechanisms of urban air quality degradation in developing countries.

The scaling relationship can also indicate the urbanization stage of different areas. Dramatic urbanization expansion has been taking place throughout the world since 1970s, and the stage of urbanization is various among continents and countries (Vitousek et al., 1997). Large cities in Europe and North America have already done the rapid urbanization, and more than $90 \%$ of the world's urbanization occurred in the developing countries in recent decades. Nevertheless, the urbanization stage differs in/among 


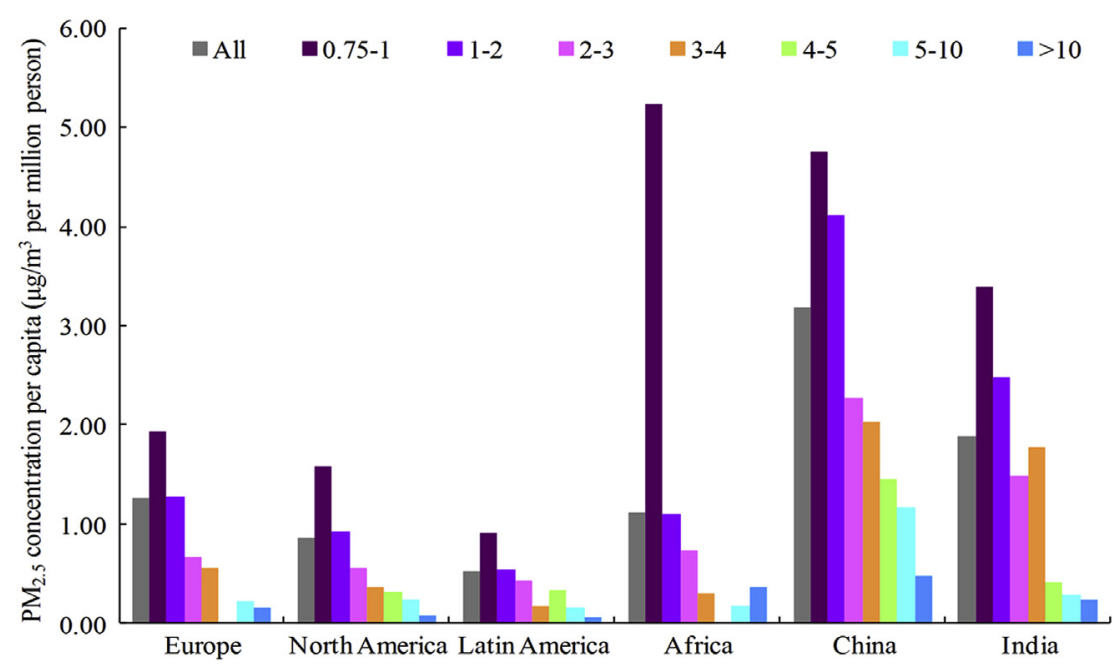

Fig. 4. $\mathrm{PM}_{2.5}$ concentration per capita at large cities of different areas.

developing countries. Latin America has similar urbanization level as developed countries, but China and India have been undergoing the most rapid urbanization. The results revealed in this work shows that large cities in China and India are suffering from the most severe air pollution, indicating the most rapid urbanization occurs with substantial costs. Moreover, large cities in Latin America have similar scaling relationship with developed countries', although their $\mathrm{PM}_{2.5}$ concentration was still higher than those in Europe and North America, indicating slow urbanization occurs.

Potential urban population's contribution to $\mathrm{PM}_{2.5}$ contribution may indicate the period of the strongest negative impact of urbanization on environment pollution (Grimm et al., 2008; Kan et al., 2012). It indicates that better policy on urbanization and air pollution prevention should emphasize groups of large cities with relative small size, rather than a few megacities, to minimize the negative impact of urbanization on air pollution. For instance, strict environmental prevention should be taken at large cities with population less than 1 million, 2 million, and 4 million in Africa, China and India, respectively.

Many developing countries have experienced rapid urbanization with negative impact on urban air quality. The scaling relationship obtained in this work would suggest a better urbanization strategy for better protecting urban air quality without limiting urban economic development. Moreover, the result may suggest an optimum city size in a country. One of the typical cases is China, where is urbanizing at an unprecedented rate. In 2013, Chinese central government released the National New-type Urbanization Plan that sets targets for China's urban population fraction to rise by $1 \%$ per year to reach $60 \%$ by 2020 , and will encourages the development of cities with small to medium size but control the numbers of megacities (Bai et al., 2014). The plan covers almost every conceivable aspect of urbanization, in which the sustainable development and prevention of environmental degradation and strategies for environmental improvement plays a critical role. Our results revealed that air quality in small and medium cities were degenerating against population increase (Fig. 3), and per capita emission was also larger at small and medium large cities (Fig. 4), indicating that special concern and actions should be taken in Chinese small and medium large cities (population less than 5 million) to let city accept more population in the future. These more modestly sized cities are projected to expand and develop faster than the larger cities in the coming years. Meanwhile, suggestions based on our result also can be given to large cities at Africa and India, where special attention and environmental protection actions should be taken at cities with population more than 5 million.

In order to improve the understanding of the scaling relationship that illustrated in this study, further research can be suggested as follows: Adopting long-term air quality monitoring in rapid urbanization large cities of different countries/continents to validate/ calibrate the scale law; developing new $\mathrm{PM}_{2.5}$ retrieval algorithm with higher spatiotemporal resolution images and relate detailed urban landscape and social-economic condition with it to better understand the impact of urbanization on air quality degradation.

\section{Conclusions}

Negative impacts of urbanization on air quality in large cities have aroused great concern. $\mathrm{PM}_{2.5}$ as a major cause of degraded air quality, is released directly or indirectly from many sources, including dust, fuel combustion, transportation, and industrial processes. Understanding the effect of urbanization on $\mathrm{PM}_{2.5}$ concentration therefore is essential to suggest improvements in urban development and may result in an optimum city size. We utilize the distribution of $\mathrm{PM}_{2.5}$ concentration and population in large cities at the global scale to illustrate the relationship between urbanization and urban air quality. Conclusions followed:

1) The relationship varies greatly among continents and countries. In particular, large cities in North America, Europe, and Latin America have better air quality than those in Asia and Africa; large cities in developed countries have better air quality than those in developing countries', with large cities in China and India having the worst air quality.

2) Urban population size increase has different relationships to $\mathrm{PM}_{2.5}$ concentration in large cities of different continents or countries. In particular, $\mathrm{PM}_{2.5}$ concentration in large cities in North America, Europe, and Latin America showed little fluctuation or a small increasing trend, but those in Africa and India represent a " $U$ " type relationship, with significant increase in large cities with population $>5$ million (Africa: $\mathrm{R}^{2}=0.71$, $\mathrm{P}<0.05$; India, $\left.\mathrm{R}^{2}=0.54, \mathrm{P}<0.05\right)$. In China, however, an inverse "U" type relationship between $\mathrm{PM}_{2.5}$ concentration and urban population is observed $\left(\mathrm{R}^{2}=0.90, \mathrm{P}<0.05\right)$, and a significant decreasing trend is observed in large cities with population $>5$ million $\left(\mathrm{R}^{2}=0.35, \mathrm{P}<0.05\right)$. 
3) The potential contribution of population to $\mathrm{PM}_{2.5}$ concentration was higher in the large cities in China and India, but lower in other large cities, except Africa, where there was the largest potential contribution in large cities with population from 0.75 to 1 million.

\section{Acknowledgment}

This research was a part of project "Spatiotemporal Pattern of Urbanized Regions' Particulate Matter (PMx) and Its Relationship with Landscape Pattern" supported by Natural Science Foundation of China (NSFC41301199). Moreover, the research also received financial support from Scientific Research Foundation for the Returned Overseas Chinese Scholars, State Education Ministry. Our appreciation also goes to Aaron van Donkelaar for providing the global annual mean satellite-derived $\mathrm{PM}_{2.5}, 2001-2006$, and $\mathrm{A}$. Schneider for providing the global maps of urban extent from satellite data.

\section{References}

Bai, X., Shi, P., Liu, Y., 2014. Realizing China's urban dream. Nature 509, 158-160. Fenger, J., 2009. Air pollution in the last 50 years - from local to global. Atmos. Environ. 43, 13-22.

Grimm, N., Faeth, S., Golubiewski, N., Redman, C., Wu, J., Bai, X., Briggs, J., 2008 Global change and the ecology of cities. Science 319, 756-760.

Han, L., Zhou, W., Li, W., Li, L., 2014. Impact of urbanization level on urban air quality: a case of fine particles $\left(\mathrm{PM}_{2.5}\right)$ in Chinese cities. Environ. Pollut. 194, 163-170.

Han, L., Zhou, W., Li, W., 2015a. City as a major source area of fine particulate $\left(\mathrm{PM}_{2.5}\right)$ in China. Environ. Pollut. 206, 183-187.

Han, L., Zhou, W., Li, W., 2015b. Increasing impact of urban fine particles $\left(\mathrm{PM}_{2.5}\right)$ on areas surrounding Chinese cities. Sci. Rep. 5, 12467.

Kan, H., Chen, R., Tong, S., 2012. Ambient air pollution, climate change, and population health in China. Environ. Int. 42, 10-19.

Lamsal, L.N., Martin, R.V., Parrish, D.D., Krotkov, N.A., 2013. Scaling relationship for $\mathrm{NO}_{2}$ pollution and urban population size: a satellite perspective. Environ. Sci. Technol. 47, 7855-7861.

Mayer, H., 1999. Air pollution in cities. Atmos. Environ. 33, 4029-4037.

National Academy of Engineering and National Research Council, 2007. Energy Futures and Urban Air Pollution: Challenges for China and the United States. The National Academies Press, Washington, DC.

Population Division, 2012. Department of Economic and Social Affairs, United Nations. World Urbanization Prospects: the 2011 Revision; Population Division. Department of Economic and Social Affairs, United Nations: New York.

Schneider, A., Friedl, M., Potere, D., 2009. A new map of global urban extent from MODIS satellite data. Environ. Res. Lett. 4 article 044003.

Schneider, A., Friedl, M., Potere, D., 2010. Monitoring urban areas globally using MODIS 500m data: new methods and datasets based on 'urban ecoregions'. Remote Sens. Environ. 114, 1733-1746.

Soubbotina, T., 2004. Beyond Economic Growth: an Introduction to Sustainable Development. The International Bank for Reconstruction and Development/The World Bank, Washington, D.C., USA.

van Donkelaar, A., Martin, R., Brauer, M., Kahn, R., Levy, R., Verduzco, C. Villeneuve, P., 2010. Global estimates of ambient fine particulate matter concentrations from satellite-based aerosol optical depth: development and application. Environ. Health Perspect. 118, 847-855.

Vitousek, P., Mooney, H., Lubchenco, J., Melillo, J., 1997. Human domination of earth's ecosystems. Science 277, 494-499. 